\title{
Stochastic porous media equation and self-organized criticality
}

\author{
Viorel Barbu, \\ Institute of Mathematics "Octav Mayer", Iasi, Romania, \\ Giuseppe Da Prato, \\ Scuola Normale Superiore di Pisa, Italy \\ and \\ Michael Röckner \\ Faculty of Mathematics, University of Bielefeld, Germany \\ and \\ Department of Mathematics and Statistics, Purdue University, \\ U. S. A.
}

May 31, 2018

\begin{abstract}
The existence and uniqueness of nonnegative strong solutions for stochastic porous media equations with noncoercive monotone diffusivity function and Wiener forcing term is proven. The finite time extinction of solutions with high probability is also proven in $1-D$. The results are relevant for self-organized critical behaviour of stochastic nonlinear diffusion equations with critical states.
\end{abstract}

AMS subject Classification 2000: 76S05, 60H15.

Key words: nonlinear stochastic diffusion equation, Brownian motion, maximal monotone operator, self-organized criticality.

\section{Introduction}

The phenomenon of self-organized criticality is widely studied in Physics from different perspectives. (We refer to [1], 2], [18], 22], [9], 15], [17, [12], 
[8] [13], 10], 16], 14] for various studies). Roughly speaking it is the property of systems to have a critical point as attractor.

The purpose of this paper is to analyze this phenomenon in the frame work of stochastic evolution equations. To the best of our knowledge this is the first time this is done in the presence of a stochastic force and in such generality in a mathematically strict way. Let us introduce our framework.

Let $\mathscr{O}$ be an open bounded domain of $\mathbb{R}^{d}, d=1,2,3$, with smooth boundary $\partial \mathscr{O}$. We shall study here the nonlinear stochastic diffusion equation,

$$
\left\{\begin{array}{l}
d X(t)-\Delta \Psi(X(t)) d t \ni \sigma(X(t)) d W(t), \quad \text { in }(0, \infty) \times \mathscr{O}, \\
\Psi(X(t)) \ni 0, \quad \text { on }(0, \infty) \times \partial \mathscr{O} \\
X(0, x)=x \quad \text { on } \mathscr{O}
\end{array}\right.
$$

where $x$ is an initial datum and $\Psi: \mathbb{R} \rightarrow 2^{\mathbb{R}}$ is a maximal monotone (possibly multivalued) graph with polynomial growth and random forcing term

$$
\sigma(X) d W=\sum_{k=1}^{\infty} \mu_{k} X d \beta_{k} e_{k}, \quad t \geq 0,
$$

where $\left\{e_{k}\right\}$ is an orthonormal basis in $L^{2}(\mathscr{O}),\left\{\mu_{k}\right\}$ is a sequence of positive numbers and $\left\{\beta_{k}\right\}$ a sequence of independent standard Brownian motions on a filtered probability space $\left(\Omega, \mathscr{F},\left\{\mathscr{F}_{t}\right\}_{t \geq 0}, \mathbb{P}\right)$.

We note that $\sigma(X)$ is defined by

$$
\sigma(X) h=\sum_{k=1}^{\infty} \mu_{k} X\left\langle h, e_{k}\right\rangle_{2} e_{k}, \quad \forall h \in L^{2}(\mathscr{O}),
$$

where $\langle\cdot, \cdot\rangle_{2}$ is the scalar product in $L^{2}(\mathscr{O})$.

The equation models the dynamics of flows in porous media and more generally the phase transition (including melting and solidification processes) in the presence of a random forcing term $\sigma(X) d W$.

Existence for stochastic equations of the form (1.1) with additive and multiplicative noise was studied in [6] under the main assumption that $\Psi$ is monotonically increasing, continuous and such that

$$
\left\{\begin{array}{l}
\Psi(0)=0, \Psi^{\prime}(r) \leq \alpha_{1}|r|^{m-1}+\alpha_{2}, \quad \forall r \in \mathbb{R}, \\
\int_{0}^{r} \Psi(s) d s \geq \alpha_{3}|r|^{m+1}+\alpha_{4}, \quad \forall r \in \mathbb{R},
\end{array}\right.
$$


where $\alpha_{1} \geq 0, \alpha_{3}>0, \alpha_{2}, \alpha_{4} \geq 0$ and $m \geq 1$. (See also [7] and [21] for general growth conditions on $\Psi$.)

Here we shall study equation (1.1) under the following assumptions.

Hypothesis $1.1 \quad$ (i) $\Psi$ is a maximal monotone multivalued function from $\mathbb{R}$ into $\mathbb{R}$ such that $0 \in \Psi(0)$.

(ii) There exist $C>0$ and $m \geq 1$ such that

$$
\sup \{|\theta|: \theta \in \Psi(r)\} \leq C\left(1+|r|^{m}\right), \quad \forall r \in \mathbb{R} .
$$

(iii) The sequence $\left\{\mu_{k}\right\}$ is such that

$$
\sum_{k=1}^{\infty} \mu_{k}^{2} \lambda_{k}^{2}<+\infty
$$

where $\lambda_{k}$ are the eigenvalues of the Laplace operator $-\Delta$ in $\mathscr{O}$ with Dirichlet boundary conditions.

We recall that the domain of $\Delta$ is $H^{2}(\mathscr{O}) \cap H_{0}^{1}(\mathscr{O})$.

Since for $x \in H^{-1}(\mathscr{O})$

$$
\left|x e_{k}\right|_{-1}^{2} \leq C_{1}\left|e_{k}\right|_{H^{2}(\mathscr{O})}^{2}|x|_{-1}^{2} \leq C_{1} \lambda_{k}^{2}|x|_{-1}^{2}
$$

and hence

$$
\|\sigma(x)\|_{L_{2}\left(L^{2}(\mathscr{O}), H^{-1}(\mathscr{O})\right)}^{2}=\sum_{k=1}^{\infty} \mu_{k}^{2}\left|x e_{k}\right|_{-1}^{2} \leq C_{1} \sum_{k=1}^{\infty} \mu_{k}^{2} \lambda_{k}^{2}|x|_{-1}^{2},
$$

it follows by (iii) that $\sigma(x) \in L_{2}\left(L^{2}(\mathscr{O}), H^{-1}(\mathscr{O})\right)$ (the space of all HilbertSchmidt operators from $L^{2}(\mathscr{O})$ into $H^{-1}(\mathscr{O})$ ) and that it is Lipschitz continuous from $H^{-1}(\mathscr{O})$ into $L_{2}\left(L^{2}(\mathscr{O}), H^{-1}(\mathscr{O})\right)$. Under these assumptions we shall prove that if $x \in L^{p}(\mathscr{O}), p \geq \max \{2 m, 4\}$, then there is a unique strong solution to equation (1.1) which is nonnegative if so is the initial data $x$. With respect to the situation considered in [7], in the present case one does not assume that the range of $\Psi$ is all of $\mathbb{R}$, which is quite unusual for porous media equations. Also Hypothesis 1.1(i) allows monotonically increasing functions $\Psi$ with a finite number of discontinuities (jumps), $r_{1}, \ldots, r_{N}$. One must, of course, fill the jumps by taking $\Psi\left(r_{j}\right)=\left[\Psi\left(r_{j}+0\right), \Psi\left(r_{j}-0\right)\right], j=r_{1}, \ldots, r_{N}$. 
It should be mentioned that several physical problems with free boundary and with phase transition can be put into this functional setting. For instance if

$$
\Psi(x)=\left\{\begin{array}{l}
\alpha_{1}(x-a), \quad \text { for } x<a \\
{[0, \rho], \text { for } x=a} \\
\alpha_{2}(x-a)+\rho, \text { for } x>a,
\end{array}\right.
$$

with $a, \rho, \alpha_{1}, \alpha_{2} \in(0,+\infty)$, then (1.1) models the phase transition in porous media or in heat conduction (Stefan problem). If $\Psi(x)=\rho \operatorname{sign} x$ where $\rho>0$ and

$$
\operatorname{sign} x=\left\{\begin{array}{l}
\frac{x}{|x|}, \quad \text { if } x \neq 0 \\
{[-1,1], \quad \text { if } x=0,}
\end{array}\right.
$$

then (1.1) reduces to the nonlinear singular diffusion equation

$$
d X(t)-\rho \operatorname{div}(\delta(X(t)) \nabla X(t)) d t=\sigma(X(t)) d W(t)
$$

where $\delta$ is the Dirac measure concentrated at the origin.

Other examples such as the Heavside step function

$$
H(x)=\left\{\begin{array}{l}
0, \quad \text { if } x<0 \\
{[0,1], \quad \text { if } x=0} \\
1, \quad \text { if } x>0,
\end{array}\right.
$$

or $\Psi(x)=|x|^{\alpha} \operatorname{sign} x$ with $0<\alpha \leq 1$ also satisfy Hypothesis 1.1.

In particular the equation

$$
d X(t)-\Delta(H+\lambda)\left(X(t)-x_{c}\right) d t=\sigma\left(X(t)-x_{c}\right) d W(t),
$$

where $\lambda>0$, represents the continuous, stochastic version of the Bak, Jang, Wiesenfeld sand pile model [1]. (See [1] for a deterministic presentation of the model.) This is a diffusion problem with free boundary driven by a random forcing term proportional to $X(t)-x_{c}$ where $x_{c}$ is the critical density and $X(t)$ is the density at the moment $t$.

Taking into account the numerical simulation in 1-D (see [2]), one might expect that the time evolution of the system displays self-organized criticality, i.e. the supercritical region $\left\{X(t)>x_{c}\right\}$ is absorbed asymptotically in time by the critical one $\left\{X(t)=x_{c}\right\}$. Here we shall prove that, e.g. in all examples (1.5)-(1.7) above, this indeed takes places with high probability 
under appropriate assumptions on the parameters and more precisely that the supercritical region "vanishes" into the critical one in finite time with high probability, at least if $\mu_{k}=0$ for all $k \geq N+1$ for some $N \in \mathbb{N}$. We emphasize that this is in particular true when the noise is zero. In this case one gets an explicit bound for the time when this happens (cf. Remark 4.4 below).

The plan of this paper is the following. The main results are presented in Section 2 and are proven in Section 3. In Section 4 we prove a finite time extinction type result for solutions to (1.1) which displays a self-organized criticality behaviour.

The following notations will be used. $L^{p}(\mathscr{O}), p \geq 1$, is the usual space of $p$-integrable functions with norm denoted by $|\cdot|_{p}$. The scalar product in $L^{2}(\mathscr{O})$ and the duality induced by the pivot space $L^{2}(\mathscr{O})$ will be denoted by $\langle\cdot, \cdot\rangle_{2} . \quad H^{k}(\mathscr{O}) \subset L^{2}(\mathscr{O}), k=1,2$, are the standard Sobolev spaces on $\mathscr{O}$, while $H_{0}^{1}(\mathscr{O})$ is the subspace of $H^{1}(\mathscr{O})$ with zero trace on the boundary. For $p, q \in[1,+\infty]$ by $L_{W}^{q}\left((0, T) ; L^{p}(\Omega ; H)\right)$ ( $H$ a Hilbert space) we shall denote the space of all $q$-integrable processes $u:[0, T] \rightarrow L^{p}(\Omega ; H)$ which are adapted to the filtration $\left\{\mathscr{F}_{t}\right\}_{t \geq 0}$.

By $C_{W}\left([0, T] ; L^{2}(\Omega ; H)\right)$ we shall denote the space of all $H$-valued adapted processes which are mean square continuous. $L(H)$ denotes the space of bounded linear operators equipped with the usual norm.

In the following by $H$ we shall denote the distribution space

$$
H=H^{-1}(\mathscr{O})=\left(H_{0}^{1}(\mathscr{O})\right)^{\prime}
$$

endowed with the scalar product and norm defined by

$$
\langle u, v\rangle=\int_{\mathscr{O}} A^{-1} u(\xi) v(\xi) d \xi, \quad|u|_{-1}=\langle u, u\rangle^{1 / 2}
$$

where $A=-\Delta$ with $D(A)=H^{2}(\mathscr{O}) \cap H_{0}^{1}(\mathscr{O})$.

In terms of $A$ equation (1.1) can be formally rewritten as

$$
\left\{\begin{array}{l}
d X(t)+A \Psi(X(t)) d t \ni \sigma(X(t)) d W(t), \\
X(0, x)=x
\end{array}\right.
$$

Its exact meaning will be precised later (see Definition 2.1 below) 
It should be recalled, however, that the operator $x \rightarrow A \Psi(x)$ with the domain

$$
\left\{x \in L^{1}(\mathscr{O}) \cap H^{-1}(\mathscr{O}): \text { there is } \eta \in H_{0}^{1}(\mathscr{O}), \eta \in \Psi(x) \text { a.e. in } \mathscr{O}\right\}
$$

is maximal monotone in $H:=H^{-1}(\mathscr{O})$ (see e.g. [3]) and so the distribution space $H$ offers the natural functional setting for the porous media equation (1.1) or its abstract form (1.8). However, the general existence theory of infinite dimensional stochastic equations in Hilbert space with nonlinear maximal monotone operators (see [11], [20]) is not applicable in the present case and so a direct approach must be used.

Fnally, in this paper we use the same letter $C$ for several different positive constants arising in chains of estimates.

\section{Existence, uniqueness and positivity}

Definition 2.1 Let $x \in H$. An $H$-valued continuous $\mathscr{F}_{t}$-adapted process $X=X(t, x)$ is called a solution to (1.1) (equivalently (1.8)) on $[0, T]$ if

$$
X \in L^{p}(\Omega \times(0, T) \times \mathscr{O}) \cap L^{2}\left(0, T ; L^{2}(\Omega, H)\right), \quad p \geq m,
$$

and there exists $\eta \in L^{p / m}(\Omega \times(0, T) \times \mathscr{O})$ such that $\mathbb{P}$-a.s.

$$
\begin{aligned}
\left\langle X(t, x), e_{j}\right\rangle_{2}= & \left\langle x, e_{j}\right\rangle_{2}+\int_{0}^{t} \int_{\mathscr{O}} \eta(s, \xi) \Delta e_{j}(\xi) d \xi d s \\
& +\sum_{k=1}^{\infty} \mu_{k} \int_{0}^{t}\left\langle X(s, x) e_{k}, e_{j}\right\rangle_{2} d \beta_{k}(s), \quad \forall j \in \mathbb{N}, t \in[0, T], \\
& \eta \in \Psi(X) \quad \text { a.e. in } \Omega \times(0, T) \times \mathscr{O} .
\end{aligned}
$$

Below for simplicity we often write $X(t)$ instead of $X(t, x)$.

From the stochastic point of view the solution $X$ given by Definition 2.1 is a strong one, but from the PDE point of view it is a solution in the sense of distributions since the boundary condition $\Psi(X) \notin 0$ on $\partial \mathscr{O}$ is satisfied in a weak sense only.

Theorem 2.2 below is the main existence result. 
Theorem 2.2 Assume that $d=1,2,3$ and that Hypothesis 1.1 holds. Then for each $x \in L^{p}(\mathscr{O}), p \geq \max \{2 m, 4\}$ there is a unique solution $X \in$ $L_{W}^{\infty}\left(0, T ; L^{p}(\Omega ; \mathscr{O})\right)$ to (1.1). Moreover, if $x$ is nonnegative a.e. in $\mathscr{O}$ then $\mathbb{P}$-a.s.

$$
X(t, x)(\xi) \geq 0, \quad \text { for a.e. }(t, \xi) \in(0, \infty) \times \mathscr{O} .
$$

As mentioned earlier, Theorem [2.2 was proven in [6] for a differentiable $\Psi$ satisfying conditions (1.2) and for $p \geq \max \{m+1,4\}$. It should be said, however, that in contrast with what happens for coercive functions $\Psi$ arising in [6], here it seems no longer possible to extend the existence result to all $x \in H^{-1}(\mathscr{O}), x \geq 0$.

\section{Proof of Theorem 2.2}

We shall consider the approximating equation

$$
\left\{\begin{array}{l}
d X_{\lambda}(t)+A\left(\Psi_{\lambda}\left(X_{\lambda}(t)\right)+\lambda X_{\lambda}(t)\right) d t=\sigma\left(X_{\lambda}(t)\right) d W(t), \\
X_{\lambda}(0, x)=x
\end{array}\right.
$$

where $\lambda>0$ and

$$
\Psi_{\lambda}(x)=\frac{1}{\lambda}\left(x-(1+\lambda \Psi)^{-1}(x)\right) \in \Psi\left((1+\lambda \Psi)^{-1}(x)\right)
$$

is the Yosida approximation of $\Psi$. We recall that $\Psi_{\lambda}$ is Lipschitzian and monotonically increasing and so $x \rightarrow \Psi_{\lambda}(x)+\lambda x$ is strictly monotonically increasing and bounded by $C_{1}\left(1+|x|^{m}\right)$ and $\left(\Psi_{\lambda}(x)+\lambda x\right) x \geq \lambda|x|^{2}$ for all $x \in \mathbb{R}$. By [6, Theorem 2.2] (applied with $m=1$ ), for each $x \in H^{-1}(\mathscr{O})$ equation (3.1) has a unique solution

$$
X_{\lambda} \in L^{2}(\Omega \times(0, T) \times \mathscr{O}) \cap L_{W}^{2}(\Omega, C([0, T] ; H))
$$

in the sense of Definition 2.1. Here as usual $C([0, T] ; H)$ is equipped with the supremum norm. Moreover, ( see e.g. [20, Theorem 4.2.5]) the following Itô formula holds

$$
\begin{aligned}
\mathbb{E}\left|X_{\lambda}(t)\right|_{-1}^{2} & +2 \mathbb{E} \int_{0}^{t} \int_{\mathscr{O}}\left(\Psi_{\lambda}\left(X_{\lambda}(s)\right)+\lambda X_{\lambda}(s)\right) X_{\lambda}(s) d \xi d s \\
& =|x|_{-1}^{2}+\sum_{k=1}^{\infty} \mu_{k}^{2} \mathbb{E} \int_{0}^{t}\left|X_{\lambda}(s) e_{k}\right|_{-1}^{2} d s .
\end{aligned}
$$


We note that since

$$
\left|X_{\lambda} e_{k}\right|_{-1} \leq C\left|e_{k}\right|_{H^{2}(\mathscr{O})}\left|X_{\lambda}\right|_{-1} \leq C \lambda_{k}\left|X_{\lambda}\right|_{-1}
$$

(cf. (1.3)) we have by Hypothesis 1.1(iii) (cf. (1.4))

$$
\sum_{k=1}^{\infty} \mu_{k}^{2} \mathbb{E} \int_{0}^{t}\left|X_{\lambda}(s) e_{k}\right|_{-1}^{2} d s \leq C \mathbb{E} \int_{0}^{t}\left|X_{\lambda}(s)\right|_{-1}^{2} d s
$$

Lemma 3.1 There exists a constant $C>0$ such that for all $p \geq 2$ and all $x \in L^{p}(\mathscr{O})$,

$$
\operatorname{ess.sup}_{t \in[0, T]} \mathbb{E}\left|X_{\lambda}(t, x)\right|_{p}^{p} \leq \exp \left(C \frac{p-1}{2}\right)|x|_{p}^{p}, \quad \forall \lambda>0 .
$$

Proof. We know from [6, Lemma 3.4] (with $m=1$ ) that as $\varepsilon \rightarrow 0$

$$
\left\{\begin{array}{l}
X_{\lambda}^{\varepsilon} \rightarrow X_{\lambda} \quad \text { strongly in } L_{W}^{\infty}\left(0, T ; L^{2}(\Omega ; H)\right) \\
X_{\lambda}^{\varepsilon} \rightarrow X_{\lambda} \quad \text { weakly star in } L_{W}^{\infty}\left(0, T ; L^{p}\left(\Omega ; L^{p}(\mathscr{O})\right)\right)
\end{array}\right.
$$

where $X_{\lambda}^{\varepsilon}$ is the solution to the approximating equation

$$
\left\{\begin{array}{l}
d X_{\lambda}^{\varepsilon}(t)+\left(A_{\lambda}\right)_{\varepsilon} X_{\lambda}^{\varepsilon}(t) d t=\sigma\left(X_{\lambda}^{\varepsilon}(t)\right) d W(t), \quad t \geq 0 \\
X_{\lambda}^{\varepsilon}(0)=x
\end{array}\right.
$$

where

$$
\left\{\begin{array}{l}
A_{\lambda} x=A\left(\Psi_{\lambda}(x)+\lambda x\right)=-\Delta\left(\Psi_{\lambda}(x)+\lambda x\right) \\
D\left(A_{\lambda}\right)=\left\{x \in H \cap L^{1}(\mathscr{O}): \Psi_{\lambda}(x)+\lambda x \in H_{0}^{1}(\mathscr{O})\right\}
\end{array}\right.
$$

and $\left(A_{\lambda}\right)_{\varepsilon}$ is the Yosida approximation of $A_{\lambda}$,

$$
\left(A_{\lambda}\right)_{\varepsilon}=\frac{1}{\varepsilon}\left(I-\left(I+\varepsilon A_{\lambda}\right)^{-1}\right), \quad \varepsilon>0
$$

Furthermore, by [6, Lemma 3.2] we have that $X_{\lambda}^{\varepsilon} \in L^{2}\left(\Omega ; C\left([0, T] ; L^{2}(\mathscr{O})\right)\right.$. As a matter of fact the results of [6] were proven for smooth nonlinear functions while $\Psi_{\lambda}$ is only Lipschitz; but the extension to lipschitzian functions 
$\Psi$ satisfying (1.2) is immediate. In fact, one might take a smoother approximation of $\Psi$, for instance the mollifier $\Psi_{\lambda} * \rho_{\lambda}\left(\rho_{\lambda}(r)=\frac{1}{\lambda} \rho(\lambda / r), \rho \in\right.$ $C_{0}^{\infty}(\mathbb{R}), \rho \geq 0, \int \rho d r=1$ ) which still remains monotonically increasing and has all properties of $\Psi_{\lambda}$.

Next we apply Itô's formula (3.6) for the function $\varphi(x)=\frac{1}{p}|x|_{p}^{p}$. More precisely, we first apply Itô's formula to $\varphi_{\gamma}(x)=\frac{1}{p}\left|(1+\gamma A)^{-1} x\right|_{p}^{p}, \gamma>0$, and then we let $\gamma \rightarrow 0$. We have (for details see the proof in [6, Lemma 3.5]),

$$
\begin{aligned}
& \mathbb{E} \varphi\left(X_{\lambda}^{\varepsilon}(t)\right)+\mathbb{E} \int_{0}^{t}\left\langle\left(A_{\lambda}\right)_{\varepsilon} X_{\lambda}^{\varepsilon}(s),\left|X_{\lambda}^{\varepsilon}(s)\right|^{p-2} X_{\lambda}^{\varepsilon}(s)\right\rangle_{2} d s \\
& =\varphi(x)+\frac{p-1}{2} \sum_{k=1}^{\infty} \mu_{k}^{2} \mathbb{E} \int_{0}^{t} \int_{\mathscr{O}}\left|X_{\lambda}^{\varepsilon}(s)\right|^{p-2}\left|X_{\lambda}^{\varepsilon}(s) e_{k}\right|^{2} d \xi d s d \xi \\
& \leq \varphi(x)+\frac{p-1}{2} C \mathbb{E} \int_{0}^{t} \int_{\mathscr{O}}\left|X_{\lambda}^{\varepsilon}(s)\right|^{p} d \xi d s,
\end{aligned}
$$

since by Sobolev embedding $\left|e_{k}\right|_{\infty} \leq C \lambda_{k}$ for all $k \in \mathbb{N}$. If $Y_{\lambda}^{\varepsilon}$ is the solution to the equation

$$
Y_{\lambda}^{\varepsilon}-\varepsilon \Delta\left(\Psi_{\lambda}\left(Y_{\lambda}^{\varepsilon}\right)+\lambda Y_{\lambda}^{\varepsilon}\right)=X_{\lambda}^{\varepsilon}, \quad \Psi_{\lambda}\left(Y_{\lambda}^{\varepsilon}\right)+\lambda Y_{\lambda}^{\varepsilon} \in H_{0}^{1}(\mathscr{O}),
$$

then (see [6, (3.25)]) $\left|Y_{\lambda}^{\varepsilon}\right|_{p} \leq\left|X_{\lambda}^{\varepsilon}\right|_{p}$ and therefore

$$
\left\langle\left(A_{\lambda}\right)_{\varepsilon} X_{\lambda}^{\varepsilon},\left|X_{\lambda}^{\varepsilon}\right|^{p-2} X_{\lambda}^{\varepsilon}\right\rangle_{2}=\frac{1}{\varepsilon}\left\langle X_{\lambda}^{\varepsilon}-Y_{\lambda}^{\varepsilon},\left|X_{\lambda}^{\varepsilon}\right|^{p-2} X_{\lambda}^{\varepsilon}\right\rangle_{2} \geq 0
$$

Then by (3.7) it follows, via Gronwall's lemma, that

$$
\mathbb{E}\left|X_{\lambda}^{\varepsilon}(t)\right|_{p}^{p} \leq|x|_{p}^{p} \exp \left(C \frac{p-1}{2}\right),
$$

where $C$ is independent of $x, \lambda$ and $t$. Now one obtains (3.4) by letting $\varepsilon$ tend to 0 and taking into account (3.5).

From now on let us assume that $p \geq \max \{4,2 m\}$ and $x \in L^{p}(\mathscr{O})$. From Lemma 3.1 it follows that for a subsequence $\{\lambda\} \rightarrow 0$ we have

$$
\left\{\begin{aligned}
X_{\lambda} \rightarrow X \quad & \text { weakly in } L^{p}(\Omega \times(0, T) \times \mathscr{O}), \\
& \text { and weakly star in in } L^{\infty}\left(0, T ; L^{p}\left(\Omega ; L^{p}(\mathscr{O})\right)\right), \\
\Psi_{\lambda}\left(X_{\lambda}\right) \rightarrow \eta \quad \text { weakly in } L^{p / m}(\Omega \times(0, T) \times \mathscr{O}), & \\
& \text { in particular in } L^{2}(\Omega \times(0, T) \times \mathscr{O}),
\end{aligned}\right.
$$


because by Hypothesis( $i i)$,

$$
\left|\Psi_{\lambda}(x)\right| \leq\left|\Psi^{0}(x)\right| \leq C\left(1+|x|^{m}\right), \quad \forall x \in \mathbb{R} .
$$

( $\Psi^{0}$ is the minimal section of $\Psi$ ). By(3.4) we have for $\lambda \rightarrow 0$

$$
\lambda X_{\lambda} \rightarrow 0 \quad \text { strongly in } L^{p}(\Omega \times(0, T) \times \mathscr{O}) .
$$

Clearly $X$ and $\eta$ are adapted processes. On the other hand, we have

$$
\begin{aligned}
& d\left(X_{\lambda}(t)-X_{\mu}(t)\right)-\Delta\left(\Psi_{\lambda}\left(X_{\lambda}(t)\right)-\Psi_{\mu}\left(X_{\mu}(t)\right)+\lambda X_{\lambda}(t)-\mu X_{\mu}(t)\right) d t \\
& =\left(\sigma\left(X_{\lambda}(t)\right)-\sigma\left(X_{\mu}(t)\right)\right) d W(t)
\end{aligned}
$$

and therefore once again applying Itô's formula (cf. (3.2)) we obtain for $\alpha>0, t \in[0, T]$,

$$
\begin{aligned}
& \left.\frac{1}{2} \mid X_{\lambda}(t)-X_{\mu}(t)\right)\left.\right|_{-1} ^{2} e^{-\alpha t} \\
& +\int_{0}^{t} \int_{\mathscr{O}}\left[\left(\Psi_{\lambda}\left(X_{\lambda}(s)\right)-\Psi_{\mu}\left(X_{\mu}(s)\right)\left(\lambda \Psi_{\lambda}\left(X_{\lambda}(s)\right)-\mu \Psi_{\mu}\left(X_{\mu}(s)\right)\right)\right.\right. \\
& \left.\quad+\left(\lambda X_{\lambda}(s)-\mu X_{\mu}(s)\right)\left(X_{\lambda}(s)-X_{\mu}(s)\right)\right] e^{-\alpha s} d \xi d s \\
& \leq\left(C \sum_{k=1}^{\infty} \mu_{k}^{2} \lambda_{k}^{2}-\frac{1}{2} \alpha\right) \int_{0}^{t}\left|X_{\lambda}\left(s-X_{\mu}(s)\right)\right|_{-1}^{2} e^{-\alpha s} d s+M_{\lambda, \mu}(t), \quad \forall \lambda, \mu>0,
\end{aligned}
$$

where

$$
M_{\lambda, \mu}(t):=\int_{0}^{t} e^{-\alpha s}\left\langle X_{\lambda}(s)-X_{\mu}(s), \sigma\left(X_{\lambda}(s)-X_{\mu}(s)\right) d W(s)\right\rangle_{2}
$$

is a real local valued martingale. To derive (3.10) we used that $x=\lambda \Psi_{\lambda}(x)+$ $(1+\lambda \Psi)^{-1}(x)$ and thus for all $x, y \in \mathbb{R}$

$$
\begin{aligned}
\left(\Psi_{\lambda}(x)-\Psi_{\mu}(y)\right)(x-y)= & {\left[\Psi_{\lambda}(x)-\Psi_{\mu}(y)\right]\left[(1+\lambda \Psi)^{-1}(x)-(1+\mu \Psi)^{-1}(y)\right] } \\
& +\left[\Psi_{\lambda}(x)-\Psi_{\mu}(y)\right]\left[\lambda \Psi_{\lambda}(x)-\mu \Psi_{\mu}(y)\right]
\end{aligned}
$$


and that the first summand on the right hand side is nonnegative because $\Psi$ is monotonically increasing and $\Psi_{\lambda}(x) \in \Psi\left((1+\lambda \Psi)^{-1}(x)\right)$. Hence for $\alpha>0$ large enough we obtain for all $\lambda, \mu \in(0,1)$ and $t \in[0, T]$

$$
\begin{aligned}
& \left.\frac{1}{2} \mid X_{\lambda}(t)-X_{\mu}(t)\right)\left.\right|_{-1} ^{2} e^{-\alpha t} \\
& \leq C \max \{\lambda, \mu\} \int_{0}^{t} \int_{\mathscr{O}}\left(\left|\Psi_{\lambda}\left(X_{\lambda}(s)\right)\right|^{2}+\left|X_{\lambda}(s)\right|^{2}+\left|\Psi_{\mu}\left(X_{\mu}(s)\right)\right|^{2}\right. \\
& \left.\quad+\left|X_{\mu}(s)\right|^{2}\right) e^{-\alpha s} d \xi d s+M_{\lambda, \mu}(t) .
\end{aligned}
$$

Hence by the Burkholder-Davis-Gundy inequality (for $p=1$ ) we get for all $\lambda, \mu \in(0,1), r \in[0, T]$,

$$
\begin{aligned}
& \left.\frac{1}{2} \mathbb{E} \sup _{t \in[0, r]} \mid X_{\lambda}(t)-X_{\mu}(t)\right)\left.\right|_{-1} ^{2} e^{-\alpha t} \\
& \leq C \max \{\lambda, \mu\} \mathbb{E} \int_{0}^{r} \int_{\mathscr{O}}\left(\left|\Psi_{\lambda}\left(X_{\lambda}(s)\right)\right|^{2}+\left|X_{\lambda}(s)\right|^{2}+\left|\Psi_{\mu}\left(X_{\mu}(s)\right)\right|^{2}\right. \\
& \left.+\left|X_{\mu}(s)\right|^{2}\right) e^{-\alpha s} d \xi d s+C \mathbb{E}\left(\int_{0}^{r}\left|X_{\lambda}(s)-X_{\mu}(s)\right|_{-1}^{4} e^{-2 \alpha s} d s\right)^{1 / 2} .
\end{aligned}
$$

But

$$
\begin{aligned}
& \mathbb{E}\left(\int_{0}^{r}\left|X_{\lambda}(s)-X_{\mu}(s)\right|_{-1}^{4} e^{-2 \alpha s} d s\right)^{1 / 2} \\
& \left.\leq \mathbb{E} \sup _{s \in[0, r]} \mid X_{\lambda}(s)-X_{\mu}(s)\right)\left.\right|_{-1} e^{-\frac{\alpha}{2} s}\left(\int_{0}^{r}\left|X_{\lambda}(s)-X_{\mu}(s)\right|_{-1}^{2} e^{-\alpha s} d s\right)^{1 / 2} \\
& \left.\leq \frac{1}{4} \mathbb{E} \sup _{s \in[0, r]} \mid X_{\lambda}(s)-X_{\mu}(s)\right)\left.\right|_{-1} ^{2} e^{-\alpha s}+C \mathbb{E} \int_{0}^{r}\left|X_{\lambda}(s)-X_{\mu}(s)\right|_{-1}^{2} e^{-\alpha s} d s .
\end{aligned}
$$

Taking into account that by Hypothesis 1.1(ii)

$$
\left|\Psi_{\lambda}\left(X_{\lambda}\right)\right| \leq C\left(1+\left|X_{\lambda}\right|^{m}\right), \quad \forall \lambda>0
$$


and that by (3.4) $\left\{X_{\lambda}\right\}$ is bounded in $L^{p}(\Omega \times(0, T) \times \mathscr{O})$ for $p \geq \max \{4,2 m\}$, we infer by (3.12), (3.13) and Gronwall's lemma that $\left\{X_{\lambda}\right\}$ is a Cauchy net in $L^{2}(\Omega ; C([0, T] ; H))$ Hence for $\lambda \rightarrow 0$

$$
X_{\lambda} \rightarrow X \quad \text { in } L^{2}(\Omega ; C([0, T] ; H)) .
$$

In order to complete the proof of the existence part of Theorem 2.2 it suffices to show that

$$
\eta(\omega, t, \xi) \in \Psi(X(\omega, t, \xi)) \text { a.e in } \Omega \times(0, T) \times \mathscr{O} .
$$

Since the operator

$L^{p}(\Omega \times(0, T) \times \mathscr{O}) \rightarrow L^{\frac{p}{m}}(\Omega \times(0, T) \times \mathscr{O}) \subset L^{\frac{p}{p-1}}(\Omega \times(0, T) \times \mathscr{O}), \quad X \rightarrow \Psi(X)$, in the duality pair

$$
\left(L^{p}(\Omega \times(0, T) \times \mathscr{O}), L^{p}(\Omega \times(0, T) \times \mathscr{O})^{\prime}=L^{\frac{p}{p-1}}(\Omega \times(0, T) \times \mathscr{O})\right),
$$

is maximal monotone, it suffices to show that (see e.g. [3])

$$
\liminf _{\lambda \rightarrow 0} \mathbb{E} \int_{0}^{T} \int_{\mathscr{O}} \Psi_{\lambda}\left(X_{\lambda}\right) X_{\lambda} d \xi d t \leq \mathbb{E} \int_{0}^{T} \int_{\mathscr{O}} \eta X d \xi d t .
$$

To prove (3.16) we first note that by (3.2) we have

$$
\begin{aligned}
& \liminf _{\lambda \rightarrow 0} \mathbb{E} \int_{0}^{T} \int_{\mathscr{O}} \Psi_{\lambda}\left(X_{\lambda}\right) X_{\lambda} d \xi d t+\frac{1}{2} \mathbb{E}|X(t)|_{-1}^{2} \\
& =\frac{1}{2}|x|_{-1}^{2}+\frac{1}{2} \sum_{k=1}^{\infty} \mu_{k}^{2} \mathbb{E} \int_{0}^{t}\left|X(s) e_{k}\right|_{-1}^{2} d s,
\end{aligned}
$$

because by (1.3), $\left|\left(X_{\lambda}-X\right) e_{k}\right|_{-1} \leq C \lambda_{k}\left|X_{\lambda}-X\right|_{-1}$ and so by Hypothesis 1.1(iii)

$$
\lim _{\lambda \rightarrow 0} \sum_{k=1}^{\infty} \mu_{k}^{2} \mathbb{E} \int_{0}^{t}\left|X_{\lambda}(s) e_{k}\right|_{-1}^{2} d s=\sum_{k=1}^{\infty} \mu_{k}^{2} \mathbb{E} \int_{0}^{t}\left|X(s) e_{k}\right|_{-1}^{2} d s .
$$

Next letting $\lambda$ tend to zero in (3.1) and using (3.8) we see that $\mathbb{P}$-a.s., for all $t \in[0, T]$,

$$
\left\langle X(t), e_{j}\right\rangle_{2}=\left\langle x, e_{j}\right\rangle_{2}+\int_{0}^{t}\left\langle\eta(s), \Delta e_{j}\right\rangle_{2} d s+\sum_{k=1}^{\infty} \mu_{k} \int_{0}^{t}\left\langle X(s) e_{k}, e_{j}\right\rangle_{2} d \beta_{k}(s) .
$$


Note that by continuity the $\mathbb{P}$-zero set does not depend on $t \in[0, T]$, since

$$
\sum_{k=1}^{\infty} \mu_{k} \int_{0}^{t}\left\langle X(s) e_{k}, e_{j}\right\rangle_{2} d \beta_{k}(s)=\int_{0}^{t}\left\langle e_{j}, \sigma(X(s)) d W(s)\right\rangle_{2} .
$$

In order to get (3.18) we have used the fact that by (3.14) we have

$$
\begin{aligned}
& \mathbb{E}\left|\int_{0}^{t}\left\langle X_{\lambda}(s) e_{k}, e_{j}\right\rangle_{2} d \beta_{k}(s) d s-\int_{0}^{t}\left\langle X(s) e_{k}, e_{j}\right\rangle_{2} d \beta_{k}(s) d s\right|^{2} \\
& =\mathbb{E} \int_{0}^{t}\left\langle\left(X_{\lambda}(s)-X(s)\right) e_{k}, e_{j}\right\rangle_{2}^{2} d s \leq C \lambda_{j}^{2} \lambda_{k}^{2} T\left|X_{\lambda}-X\right|_{L^{2}(\Omega, C([0, T] ; H))}^{2}
\end{aligned}
$$

and therefore

$$
\lim _{\lambda \rightarrow 0} \sum_{k=1}^{\infty} \mu_{k} \int_{0}^{t}\left\langle X_{\lambda}(s) e_{k}, e_{j}\right\rangle_{2} d \beta_{k} d s=\sum_{k=1}^{\infty} \mu_{k} \int_{0}^{t}\left\langle X(s) e_{k}, e_{j}\right\rangle_{2} d \beta_{k} d s .
$$

Therefore (3.18) follows and this yields, via Itô's formula (applied to $\left\langle X(t), e_{j}\right\rangle_{2}^{2}$, $t \in[0, T])$ and summation over $j$ that

$$
\begin{aligned}
& \frac{1}{2} \mathbb{E}|X(t)|_{-1}^{2}+\mathbb{E} \int_{0}^{t} \int_{\mathscr{O}} \eta X d \xi d s \\
& =\frac{1}{2} \mathbb{E}|x|_{-1}^{2}+\frac{1}{2} \sum_{k=1}^{\infty} \mu_{k}^{2} \mathbb{E} \int_{0}^{t}\left|X(s) e_{k}\right|_{-1}^{2} d s, \forall t \in[0, T] .
\end{aligned}
$$

Comparing (3.17) and (3.19) we get (3.16). Hence $X$ is a solution to (1.1) as claimed.

To prove uniquenss we take two solutions $X^{(1)}$ and $X^{(2)}$ with corresponding $\eta^{(1)}$ and $\eta^{(2)}$. Repeating the argument above we obtain

$$
\begin{aligned}
& \frac{1}{2} \mathbb{E}\left|X^{(1)}(t)-X^{(2)}(t)\right|_{-1}^{2} \\
& +\mathbb{E} \int_{0}^{t} \int_{\mathscr{O}}\left(\eta^{(1)}(s)-\eta^{(2)}(s)\right)\left(X^{(1)}(s)-X^{(2)}(s)\right) d \xi d s \\
& =\frac{1}{2} \sum_{k=1}^{\infty} \mu_{k}^{2} \mathbb{E} \int_{0}^{t}\left|\left(X^{(1)}(s)-X^{(2)}(s)\right) e_{k}\right|_{-1}^{2} d s, \quad \forall t \in[0, T] .
\end{aligned}
$$


Since, because $\Psi$ is monotone, the second term on the left is positive, by (1.3), Hpothesis 1.1(iii) this implies $X^{(1)}=X^{(2)}$ by Gronwall's lemma.

Finally, if $x \geq 0$ a.e. in $\mathscr{O}$ we know by [6, Theorem 2.2] that $X_{\lambda} \geq 0$ $\mathbb{P}$-a.s. and so by (3.14) it follows that $X \geq 0$, a.e in $\Omega \times(0, T) \times \mathscr{O}$ as desired. This completes the proof of Theorem 2.2 .

Remark 3.2 Theorem 2.2 extends to any dimension $d \geq 1$ if one modifies condition (iii) in Hypothesis 1.1] as in [6, Condition 4.1].

Remark 3.3 The existence part of Theorem 2.2 remains true for stochastic porous media equations with additive noise, i.e.

$$
d X-\Delta \Psi(X) d t=\sqrt{Q} d W(t)
$$

where $\Psi$ satisfies Hypothesis 1.1 and

$$
\sqrt{Q} d W(t)=\sum_{k=1}^{\infty} \mu_{k} e_{k} d \beta_{k}(t)
$$

with

$$
\sum_{k=1}^{\infty} \lambda_{k}^{-1} \mu_{k}^{2}<+\infty .
$$

The proof is exactly the same and so, it will be omitted.

Proposition 3.4 Let $X_{\lambda}, \lambda \in(0,1)$, be as above, $x \in L^{4}(\mathscr{O})$. Assume that $\Psi$ satisfies Hypothesis 1.1 with $m=1$ and for some $\delta>0$,

$$
(\tilde{x}-\tilde{y})(x-y) \geq \delta(x-y)^{2}, \quad \forall(x, \tilde{x}),(y, \tilde{y}) \in \Psi .
$$

Then $X_{\lambda}, X \in L_{W}^{2}\left(0, T ; L^{2}\left(\Omega, H_{0}^{1}(\mathscr{O})\right)\right)$ and

$$
\lim _{\lambda \rightarrow 0} \mathbb{E}\left|X_{\lambda}-X\right|_{L^{2}\left(0, T ; L^{2}(\mathscr{O})\right)}^{2}=0 .
$$

Proof. A simple calculation reveals that

$$
\left(\Psi_{\lambda}(x)-\Psi_{\lambda}(y)\right)(x-y) \geq \frac{\delta}{2}|x-y|^{2}, \quad \forall x, y \in \mathbb{R}
$$


for $\lambda$ sufficiently small. Then $\tilde{\Psi}_{\lambda}$ defined by $\tilde{\Psi}_{\lambda}(r):=\Psi_{\lambda}(r)-\frac{\delta}{2} r, r \in \mathbb{R}$, is increasing and so by Itô's formula we have

$$
\mathbb{E}\left|X_{\lambda}(t)\right|_{2}^{2}+\frac{\delta}{2} \mathbb{E} \int_{0}^{t}\left|X_{\lambda}(s)\right|_{H_{0}^{1}(\mathscr{O})}^{2} d s \leq C .
$$

As a matter of fact, we shall apply Itô's formula not directly to equation (3.1) but to equation (3.6) (cf. the proof of Lemma 3.1 to obtain (3.7)). Thus we get

$$
\frac{1}{2} \mathbb{E}\left|X_{\lambda}^{\varepsilon}(t)\right|_{2}^{2}+\mathbb{E} \int_{0}^{t}\left\langle\left(A_{\lambda}\right)_{\varepsilon} X_{\lambda}^{\varepsilon}(s), X_{\lambda}^{\varepsilon}(s)\right\rangle_{2} d s \leq \frac{1}{2}|x|_{2}^{2}+C \mathbb{E} \int_{0}^{t}\left|X_{\lambda}^{\varepsilon}(s)\right|_{2}^{2} d s .
$$

Next we have

$$
\left\langle\left(A_{\lambda}\right)_{\varepsilon} X_{\lambda}^{\varepsilon}, X_{\lambda}^{\varepsilon}\right\rangle_{2}=\left\langle A_{\lambda}\left(1+\varepsilon A_{\lambda}\right)^{-1} X_{\lambda}^{\varepsilon},\left(1+\varepsilon A_{\lambda}\right)^{-1} X_{\lambda}^{\varepsilon}\right\rangle_{2}+\varepsilon\left|\left(A_{\lambda}\right)_{\varepsilon} X_{\lambda}^{\varepsilon}\right|_{2}^{2} .
$$

Taking into account that $A_{\lambda}=\Delta\left(\Psi_{\lambda}+\lambda I\right)$ and that $r \rightarrow \Psi_{\lambda}(r)-\delta r / 2$ is monotonically increasing we get

$$
\left\langle\left(A_{\lambda}\right)_{\varepsilon} X_{\lambda}^{\varepsilon}, X_{\lambda}^{\varepsilon}\right\rangle_{2} \geq \frac{\delta}{2} \int_{\mathscr{O}}\left|\nabla\left(1+\varepsilon A_{\lambda}\right)^{-1} X_{\lambda}^{\varepsilon}\right|^{2} d \xi+\varepsilon\left|\left(A_{\lambda}\right)_{\varepsilon} X_{\lambda}^{\varepsilon}\right|_{2}^{2}
$$

Hence

$$
\mathbb{E} \int_{0}^{t}\left|\left(1+\varepsilon A_{\lambda}\right)^{-1} X_{\lambda}^{\varepsilon}(s)\right|_{H_{0}^{1}(\mathscr{O})}^{2} d s \leq C
$$

and letting $\varepsilon \rightarrow 0$ we get (3.22) and the first assertion (taking also into account (3.5)).

To prove the second part we note that

$$
\begin{aligned}
& d\left(X_{\lambda}-X_{\mu}\right)-\Delta\left[\tilde{\Psi}_{\lambda}\left(X_{\lambda}\right)-\tilde{\Psi}_{\mu}\left(X_{\mu}\right)+\lambda X_{\lambda}-\mu X_{\mu}+\frac{1}{2} \delta\left(X_{\lambda}-X_{\mu}\right)\right] d t \\
& =\left(\sigma\left(X_{\lambda}\right)-\sigma\left(X_{\mu}\right)\right) d W .
\end{aligned}
$$

Hence exactly the same arguments to derive (3.11) lead to

$$
\begin{gathered}
\frac{1}{2}\left|X_{\lambda}(t)-X_{\mu}(t)\right|_{-1}^{2} e^{-\alpha t}+\frac{\delta}{2} \int_{0}^{t}\left|X_{\lambda}(s)-X_{\mu}(s)\right|_{2}^{2} e^{-\alpha s} d s \\
\leq C \max \{\lambda, \mu\} \int_{0}^{t}\left(\left|\Psi_{\lambda}\left(X_{\lambda}(s)\right)\right|_{2}^{2}+\left|\Psi_{\mu}\left(X_{\mu}(s)\right)\right|_{2}^{2}\right. \\
\left.+\left|X_{\lambda}(s)\right|_{2}^{2}+\left|X_{\mu}(s)\right|_{2}^{2}\right) e^{-\alpha s} d s+M_{\lambda, \mu}(t),
\end{gathered}
$$


for $\alpha$ large enough and $\lambda, \mu \in(0,1), t \in[0, T]$. Since $m=1$, we have $\left|\Psi_{\lambda}(x)\right| \leq C(1+|x|)$ for all $x \in \mathbb{R}, \lambda \in(0,1)$, hence taking expectation we get

$$
\frac{\delta}{2} \mathbb{E} \int_{0}^{t}\left|X_{\lambda}(s)-X_{\mu}(s)\right|_{2}^{2} d s \leq C \max \{\lambda, \mu\} \mathbb{E} \int_{0}^{t}\left(\left|X_{\lambda}(s)\right|^{2}+\left|X_{\mu}(s)\right|^{2}\right) d s .
$$

By Lemma 3.1 with $p=2$ and (3.8) this implies (3.21).

Besides Hypothesis 1.1, we shall now assume the following

(iv) $\Psi(r)=\rho \operatorname{sign} r+\widetilde{\Psi}(r)$, for $r \in \mathbb{R}$, where $\rho>0$, $\widetilde{\Psi}: \mathbb{R} \rightarrow \mathbb{R}$ is Lipschitzian, $\widetilde{\Psi} \in C^{1}(\mathbb{R} \backslash\{0\})$ and for some $\delta>0$ it satisfies $\widetilde{\Psi}^{\prime}(r) \geq \delta$ for all $r \in \mathbb{R} \backslash\{0\}$.

Here the signum is defined by (1.6).

Below we shall use an approximation to $\Psi$ which is slightly different from $\Psi_{\lambda}$ defined before. Namely, below we consider

$$
\Psi_{\lambda}(r):=\rho(\operatorname{sign})_{\lambda}(r)+\widetilde{\Psi}(r), \quad r \in \mathbb{R}
$$

where $(\operatorname{sign})_{\lambda}$ is the Yosida approximation of the sign, i.e.

$$
(\operatorname{sign})_{\lambda}(r):=\left\{\begin{array}{l}
1 \text { if } r>\lambda \\
\frac{r}{\lambda} \text { if } r \in[-\lambda, \lambda] \\
-1 \quad \text { if } r<-\lambda .
\end{array}\right.
$$

We shall use the symbol $\Psi_{\lambda}$ also for this approximation and denote also by $X_{\lambda}$ the corresponding solution of (3.1). This approximation in the special case of condition (iv) is much more convenient. We emphasize that all previous results remain true for this modified approximation. The proofs are the same and some parts even simplify. We therefore shall use all previous results for $\Psi_{\lambda}$ and $X_{\lambda}$ as above without further notice.

Proposition 3.5 The solutions $X_{\lambda}$ to (3.1) and $X$ to (1.1) satisfy all conditions of Proposition 3.4 and in addition

$$
\mathbb{E} \int_{0}^{T} \int_{\mathscr{O}}\left|\nabla(\operatorname{sign})_{\lambda}\left(X_{\lambda}\right)\right|^{2} d \xi d t \leq C, \quad \forall \lambda>0
$$

and consequently $\eta \in L_{W}^{2}\left(0, T ; L^{2}\left(\Omega ; H_{0}^{1}(\mathscr{O})\right)\right.$. 
Proof. We set

$$
g_{\lambda}(r):=\int_{0}^{r}(\operatorname{sign})_{\lambda}(s) d s, \quad r \in \mathbb{R}
$$

and choose $\varphi_{\lambda} \in C^{2}(\mathbb{R})$ such that

(i) $\varphi_{\lambda}(0)=0$.

(ii) $\varphi_{\lambda}^{\prime}(r)=\frac{r}{\lambda}$ for $|r| \leq \lambda, \varphi_{\lambda}^{\prime}(r)=1+\lambda$ for $r \geq 2 \lambda, \varphi_{\lambda}^{\prime}(r)=-1-\lambda$ for $r \leq-2 \lambda$.

(iii) $0 \leq \varphi_{\lambda}^{\prime \prime}(r) \leq \frac{C}{\lambda}$ for all $r \in \mathbb{R}$.

It is easily seen that such a function exists and can be constructed simply by smoothing the function $(\operatorname{sign})_{\lambda}$. Let us denote the resulting function by $f_{\lambda}$. Then define

$$
\varphi_{\lambda}(r):=\int_{0}^{r} f_{\lambda}(s) d s, \quad r \in \mathbb{R}
$$

As mentioned above the arguments of the previous proofs extends to the present situation in order to prove that $\left\{X_{\lambda}\right\}$ is convergent to the solution $X$ to (1.1).

Now we shall apply Itô's formula to equation (3.1) (or, more exactly, to (3.6) and then let $\varepsilon \rightarrow 0$ as in the proof of Proposition 3.4) with $\Psi_{\lambda}$ defined as above and to the function $\int_{\mathscr{O}} \varphi_{\lambda}\left(X_{\lambda}\right) d \xi$.

Arguing as in the proof of Lemma 3.1 to obtain (3.7), we get (recall that $\left.X_{\lambda}(t) \in H_{0}^{1}(\mathscr{O})\right)$

$$
\begin{aligned}
& \mathbb{E} \int_{\mathscr{O}} \varphi_{\lambda}\left(X_{\lambda}(t)\right) d \xi-\mathbb{E} \int_{0}^{t}\left\langle\Delta(\operatorname{sign})_{\lambda}\left(X_{\lambda}(s)\right)+\Delta \widetilde{\Psi}\left(X_{\lambda}(s)\right), \varphi_{\lambda}^{\prime}\left(X_{\lambda}(s)\right)\right\rangle_{2} d s \\
& \leq \int_{\mathscr{O}} \varphi_{\lambda}(x) d \xi+C \sum_{k=1}^{\infty} \mu_{k}^{2} \mathbb{E} \int_{0}^{t} \int_{\mathscr{O}} \varphi_{\lambda}^{\prime \prime}\left(X_{\lambda}(s)\right)\left|X_{\lambda}(s) e_{k}\right|^{2} d \xi d s \\
& \leq \int_{\mathscr{O}} \varphi_{\lambda}(x) d \xi+4 \lambda C \sum_{k=1}^{\infty} \mu_{k}^{2} \lambda_{k}^{2} \mathbb{E} \int_{0}^{t} \int_{\mathscr{O}} 1_{\lambda}(s, \xi)\left|e_{k}\right|^{2} d \xi d s
\end{aligned}
$$

where $1_{\lambda}$ is the characteristic function of the set $\left\{(s, \xi): 0 \leq\left|X_{\lambda}(s, \xi)\right| \leq\right.$ $2 \lambda\}$. 
Concerning the first line we note that, since $\varphi_{\lambda}^{\prime}$ and $\tilde{\Psi}$ are monotonically increasing while as seen earlier $X_{\lambda}(t) \in H_{0}^{1}(\mathscr{O})$, we have by the Green formula that

$$
\left\langle\Delta \widetilde{\Psi}\left(X_{\lambda}\right), \varphi_{\lambda}^{\prime}\left(X_{\lambda}\right)\right\rangle_{2}=-\int_{\mathscr{O}} \widetilde{\Psi}^{\prime}\left(X_{\lambda}\right) \varphi_{\lambda}^{\prime \prime}\left(X_{\lambda}\right)\left|\nabla X_{\lambda}\right|^{2} d \xi \leq 0 .
$$

This yields

$$
\mathbb{E} \int_{0}^{T} \int_{\mathscr{O}}\left\langle\nabla(\operatorname{sign})_{\lambda}\left(X_{\lambda}\right), \nabla \varphi_{\lambda}^{\prime}\left(X_{\lambda}\right)\right\rangle_{2} d \xi d s \leq C, \quad \forall \lambda \in(0,1) .
$$

Taking into account that

$$
-\left\langle\Delta(\operatorname{sign})_{\lambda}\left(X_{\lambda}\right), \varphi_{\lambda}^{\prime}\left(X_{\lambda}\right)\right\rangle_{2}=\left\langle\nabla(\operatorname{sign})_{\lambda}\left(X_{\lambda}\right), \nabla \varphi_{\lambda}^{\prime}\left(X_{\lambda}\right)\right\rangle_{2} \geq 0, \quad \text { a.e. }
$$

and that $\nabla \varphi_{\lambda}^{\prime}\left(X_{\lambda}\right)=\frac{1}{\lambda} \nabla X_{\lambda}$ on $\left\{(s, \xi):\left|X_{\lambda}(s, \xi)\right|<\lambda\right\}$ we get

$$
\mathbb{E} \int_{0}^{T} \int_{\mathscr{O}}\left|\nabla(\operatorname{sign})_{\lambda}\left(X_{\lambda}\right)\right|^{2} d \xi d s \leq C, \quad \forall \lambda \in(0,1),
$$

because $\nabla(\operatorname{sign})_{\lambda}\left(X_{\lambda}\right)=\frac{1}{\lambda} \nabla\left(X_{\lambda}\right)$ if $\left.\mid X_{\lambda}\right) \mid<\lambda$ and $\nabla(\operatorname{sign})_{\lambda}\left(X_{\lambda}\right)=0$ if $\left.\mid X_{\lambda}\right) \mid \geq \lambda$.

Then we get the desired estimate and since also by (3.22)

$$
\mathbb{E} \int_{0}^{T} \int_{\mathscr{O}}\left|\nabla \widetilde{\Psi}\left(X_{\lambda}\right)\right|^{2} d \xi d s \leq C, \quad \forall \lambda \in(0,1)
$$

and $(\operatorname{sign})_{\lambda}\left(X_{\lambda}\right)+\widetilde{\Psi}\left(X_{\lambda}\right) \rightarrow \eta$ weakly in $L^{2}(\Omega \times(0, T) \times \mathscr{O})$ as $\lambda \rightarrow 0$ we infer that $\eta \in L_{W}^{2}\left(0, T ; L^{2}\left(\Omega ; H_{0}^{1}(\mathscr{O})\right)\right.$ as claimed.

\section{Extinction in finite time and self-organized criticality}

In this section we shall prove a finite extinction property for solutions of (1.1) in 1- $D$ for a special density dependent diffusion coefficient function $\Psi$. However, Lemma 4.1 below can be proved without restriction on dimension. So, for the moment we remain in our general framework.

For simplicity we choose the Wiener process

$$
W(t)=\sum_{k=1}^{N} \mu_{k} e_{k} \beta_{k}(t), \quad t \geq 0,
$$


where $N \in \mathbb{N}$.

Besides Hypothesis 1.1, we shall assume Hypothesis (iv) (see page 16), i.e.

(iv) $\Psi(r)=\rho \operatorname{sign} r+\widetilde{\Psi}(r)$, for $r \in \mathbb{R}$, where $\rho>0, \widetilde{\Psi}: \mathbb{R} \rightarrow \mathbb{R}$ is Lipschitzian, $\widetilde{\Psi} \in C^{1}(\mathbb{R} \backslash\{0\})$ and for some $\delta>0$ it satisfies $\widetilde{\Psi}^{\prime}(r) \geq \delta$ for all $r \in \mathbb{R} \backslash\{0\}$.

Here the signum is defined by (1.6).

Now let $\tau$ be the stopping time

$$
\tau=\inf \left\{t \geq 0:|X(t, x)|_{-1}=0\right\},
$$

where $X(t, x), t \geq 0$, is the solution to (1.1) given by Theorem 2.2 for $x \in$ $L^{p}(\mathscr{O}), p \geq \max \{4,2 m\}$.

Lemma 4.1 Under assumptions (i)-(iv) we have

$$
X(t, x)=0, \quad \text { for } t \geq \tau, \quad \mathbb{P} \text {-a.s.. }
$$

Proof. Set $A=-\Delta, D(A)=H^{2}(\mathscr{O}) \cap H_{0}^{1}(\mathscr{O})$. Define $\mu:[0, T] \times \Omega \rightarrow$ $C_{b}^{2}(\mathscr{O} ; \mathbb{R})$ by

$$
\mu(t):=-\sum_{k=1}^{N} \mu_{k} e_{k} \beta_{k}(t), \quad t \in[0, T],
$$

and $\tilde{\mu}:[0, T] \rightarrow C_{b}^{2}(\mathscr{O} ; \mathbb{R})$ by

$$
\tilde{\mu}:=\sum_{k=1}^{N} \mu_{k}^{2} e_{k}^{2} .
$$

Define

$$
Y(t)=e^{\mu(t)} X(t), \quad t \geq 0 .
$$

Let $D(A)$ be equipped with the graph norm of $A$ and let $D(A)^{\prime}$ be its dual space, hence

$$
D(A) \subset H_{0}^{1}(\mathscr{O}) \subset L^{2}(\mathscr{O}) \subset H^{-1}(\mathscr{O}) \subset D(A)^{\prime}
$$

It is easy to see that for all $\omega \in \Omega, t \in[0, T]$ the function $e^{\mu(t, \omega)}$ is a multiplier both in $D(A)$ and in $H$, hence $e^{\mu(t, \omega)} \Delta z \in D(A)^{\prime}$ is well defined for all $z \in L^{2}(\mathscr{O})$ and $Y(t) \in H$. 
Claim. We have

$$
Y(t)=x+\int_{0}^{t} e^{\mu(s)} \Delta \eta(s) d s-\frac{1}{2} \int_{0}^{t} \tilde{\mu} Y(s) d s, \quad t \in[0, T],
$$

where the fist integral on the right hand side is a Bochner integral in $D(A)^{\prime}$, the second by (3.8) is one in $L^{p}(\mathscr{O}) \subset L^{2}(\mathscr{O})$. In particular a posteriori the first integal is in $H$, continuous in $H$ as a function of $t \in[0, T], \mathbb{P}$-a.s.

Proof of the Claim. Let $\varphi \in D(A)$. As before we shall use $\langle\cdot, \cdot\rangle_{2}$ also for the extended dualizations with pivot space $L^{2}(\mathscr{O})$ as the ones in (4.2). Then for $t \in[0, T]$

$$
\left\langle\varphi, e^{\mu(t)} X(t)\right\rangle_{2}=\sum_{j=1}^{\infty}\left\langle e_{j}, e^{\mu(t)} \varphi\right\rangle_{2}\left\langle e_{j}, X(t)\right\rangle_{2}
$$

Furthermore, we have by Itô's formula for all $\xi \in \mathscr{O}$

$$
e^{\mu(t, \xi)}=1+\int_{0}^{t} e^{\mu(s, \xi)} d \mu(s, \xi)+\frac{1}{2} \int_{0}^{t} e^{\mu(s, \xi)} \tilde{\mu}(\xi) d s .
$$

Now fix $j \in \mathbb{N}$. Then by the stochastic Fubini Theorem

$$
\begin{aligned}
\left\langle e_{j}, e^{\mu(t)} \varphi\right\rangle_{2}= & \left\langle e_{j}, \varphi\right\rangle_{2}-\sum_{k=1}^{N} \mu_{k} \int_{0}^{t}\left\langle e_{j}, e_{k} e^{\mu(s)} \varphi\right\rangle_{2} d \beta_{k}(s) \\
& +\frac{1}{2} \int_{0}^{t}\left\langle e_{j}, \tilde{\mu} e^{\mu(s)} \varphi\right\rangle_{2} d s, \quad t \in[0, T] .
\end{aligned}
$$


By Itô's product rule and (3.18) we hence obtain

$$
\begin{aligned}
& \left\langle e_{j}, e^{\mu(t)} \varphi\right\rangle_{2}\left\langle e_{j}, X(t)\right\rangle_{2}=\left\langle e_{j}, \varphi\right\rangle_{2}\left\langle e_{j}, x\right\rangle_{2} \\
& +\int_{0}^{t}\left\langle e_{j}, e^{\mu(s)} \varphi\right\rangle_{2}\left\langle\Delta e_{j}, \eta(s)\right\rangle_{2} d s \\
& +\sum_{k=1}^{N} \mu_{k} \int_{0}^{t}\left\langle e_{j}, e^{\mu(s)} \varphi\right\rangle_{2}\left\langle e_{j}, X(s) e_{k}\right\rangle_{2} d \beta_{k}(s) \\
& +\frac{1}{2} \int_{0}^{t}\left\langle e_{j}, X(s)\right\rangle_{2}\left\langle e_{j}, \tilde{\mu} e^{\mu(s)} \varphi\right\rangle_{2} d s \\
& -\sum_{k=1}^{N} \mu_{k} \int_{0}^{t}\left\langle e_{j}, X(s)\right\rangle_{2}\left\langle e_{j}, e_{k} e^{\mu(s)} \varphi\right\rangle_{2} d \beta_{k}(s) \\
& -\sum_{k=1}^{N} \mu_{k}^{2} \int_{0}^{t}\left\langle e_{j}, e_{k} e^{\mu(s)} \varphi\right\rangle_{2}\left\langle e_{j}, X(s) e_{k}\right\rangle_{2} d \beta_{k}(s) .
\end{aligned}
$$

After summing over $j \in \mathbb{N}$ the two stochastic terms cancel and the claim follows since $\varphi \in D(A)$ was arbitrary.

Below we work for $\mathbb{P}$-a.s. $\omega \in \Omega, \omega$ fixed. Hence all constants $C$ appearing below may depend on $\omega$.

Consider the solution $X_{\lambda} \in L_{W}^{2}\left(0, T ; L^{2}\left(\Omega, H_{0}^{1}(\mathscr{O})\right)\right)$ to equation (3.1). By Proposition 3.4 we have

$$
\lim _{\lambda \rightarrow 0} \mathbb{E}\left|X_{\lambda}-X\right|_{L^{2}\left(0, T ; L^{2}(\mathscr{O})\right)}^{2}=0
$$

and $\Psi_{\lambda}\left(X_{\lambda}\right) \in L_{W}^{2}\left(0, T ; L^{2}\left(\Omega, H_{0}^{1}(\mathscr{O})\right)\right)$ because $\Psi_{\lambda}$ is Lipschitz.

On the other hand,we have as in (4.3) for $Y_{\lambda}=e^{\mu} X_{\lambda}$

$$
\frac{d Y_{\lambda}(t)}{d t}=e^{\mu(t)} \Delta \eta_{\lambda}(t)-\frac{1}{2} \tilde{\mu}(t) Y_{\lambda}(t), \quad \forall t \geq 0
$$

where

$$
\eta_{\lambda}(t)=\Psi_{\lambda}\left(X_{\lambda}(t)\right) \in H_{0}^{1}(\mathscr{O}) .
$$

It follows by (3.21) that

$$
\lim _{\lambda \rightarrow 0} \mathbb{E}\left|Y_{\lambda}-Y\right|_{L^{2}\left(0, T ; L^{2}(\mathscr{O})\right)}^{2}=0
$$


and therefore for some sequence $\lambda_{n} \rightarrow 0$

$$
\lim _{n \rightarrow \infty}\left|Y_{\lambda_{n}}-Y\right|_{L^{2}\left(0, T ; L^{2}(\mathscr{O})\right)}=0 \quad \text { a.e. on } \Omega \text {. }
$$

Below we simple write $\lambda$ instead of $\lambda_{n}$. Next we have by (4.4) that

$$
\left\langle\frac{d Y_{\lambda}(t)}{d t}, Y_{\lambda}(t)\right\rangle_{2}=\left\langle\eta_{\lambda}(t), \Delta\left(e^{\mu(t)} Y_{\lambda}(t)\right)\right\rangle_{2}-\frac{1}{2}\left\langle\tilde{\mu}(t) Y_{\lambda}(t), Y_{\lambda}(t)\right\rangle_{2} \quad \text { a.e. } t \in[0, T] .
$$

Also we have (for simplicity we take $\rho=1$ )

$$
\begin{aligned}
& \left\langle\eta_{\lambda}(t), \Delta\left(e^{\mu(t)} Y_{\lambda}(t)\right)\right\rangle_{2} \\
& =\left\langle(\operatorname{sign})_{\lambda}\left(e^{-\mu(t)} Y_{\lambda}(t)\right)+\widetilde{\Psi}\left(e^{-\mu(t)} Y_{\lambda}(t)\right), \Delta\left(e^{\mu(t)} Y_{\lambda}(t)\right)\right\rangle_{2} \\
& =-\int_{\mathscr{O}}\left(\nabla(\operatorname{sign})_{\lambda}\left(e^{-\mu(t)} Y_{\lambda}(t)\right), \nabla\left(e^{\mu(t)} Y_{\lambda}(t)\right)\right) d \xi \\
& -\int_{\mathscr{O}} \widetilde{\Psi}^{\prime}\left(e^{-\mu(t)} Y_{\lambda}(t)\right)\left(\nabla\left(e^{-\mu(t)} Y_{\lambda}(t)\right), \nabla\left(e^{\mu(t)} Y_{\lambda}(t)\right)\right) d \xi \\
& =-\frac{1}{\lambda} \int_{\mathscr{O}}\left(\left|\nabla Y_{\lambda}(t)\right|^{2}-\left|Y_{\lambda}(t)\right|^{2}|\nabla \mu(t)|^{2}\right) 1_{\lambda}(t, \xi) d \xi \\
& -\int_{\mathscr{O}} \widetilde{\Psi}^{\prime}\left(e^{-\mu(t)} Y_{\lambda}(t)\right)\left(\left|\nabla Y_{\lambda}(t)\right|^{2}-\left|Y_{\lambda}(t)\right|^{2}|\nabla \mu(t)|^{2}\right) d \xi,
\end{aligned}
$$

because for $y \in H_{0}^{1}(\mathscr{O})$

$$
\nabla(\operatorname{sign})_{\lambda}(y)=\left\{\begin{array}{l}
0, \quad \text { on }\{y \notin(-\lambda, \lambda)\}, \\
\frac{1}{\lambda} \nabla y, \quad \text { on }\{y \in(-\lambda, \lambda)\} .
\end{array}\right.
$$

(Here $1_{\lambda}$ is the characteristic function of $\left\{(\xi, t) \in \mathscr{O} \times[0, T]: \mid e^{-\mu(t, \xi)} Y_{\lambda}(t, \xi)\right) \mid<$ $\lambda\}$ and $(\cdot, \cdot)$ is the euclidean scalar product in $\mathbb{R}^{n}$.) Since $\widetilde{\Psi}^{\prime} \geq \delta$ and $\widetilde{\Psi}^{\prime} \in L^{\infty}(\mathbb{R}), \mu \in C([0, T] \times \mathscr{O})$ this yields

$$
\left\langle\eta_{\lambda}(t), \Delta\left(e^{\mu(t)} Y_{\lambda}(t)\right)\right\rangle_{2} \leq C\left(\left|Y_{\lambda}(t)\right|_{2}^{2}+\lambda\right) .
$$

Hence (4.7) and Gronwall's lemma imply

$$
\left|Y_{\lambda}(t)\right|_{2}^{2} \leq e^{C(t-s)}\left(\left|Y_{\lambda}(s)\right|_{2}^{2}+C \lambda T\right) \quad \text { a.e. } t>s .
$$


Now taking into account (4.6) and letting $\lambda \rightarrow 0$ we get

$$
|Y(t)|_{2}^{2} \leq e^{C(t-s)}|Y(s)|_{2}^{2} \quad \text { a.e. } t>s .
$$

If $Y(\cdot)$ is $L^{2}(\mathscr{O})$-continuous then (4.9) holds for all $s, t \in[0, T], t \geq s$. Taking in (4.9) $s=\tau \wedge T$ we get $Y(t)=0$ for all $t \geq \tau \wedge T$ and since $T>0$ was arbitrary for all $t \geq \tau$ as claimed. So, we have to prove that $Y$ is $L^{2}(\mathscr{O})$ continuous on $[0, T]$. For this we recall that by Proposition 3.5 we have

$$
e^{\mu} \eta \in L^{2}\left(0, T ; H_{0}^{1}(\mathscr{O})\right), \quad \mathbb{P} \text {-a.s.. }
$$

Then by equation (4.3) we have $\frac{d Y}{d t} \in L^{2}\left(0, T ; H^{-1}(\mathscr{O})\right)$ and so, since $Y \in$ $L^{2}\left(0, T ; H_{0}^{1}(\mathscr{O})\right) \mathbb{P}$-a.s. by Proposition 3.4 , by a well known interpolation result (see e.g. [3]), we conclude that $Y \in C\left([0, T] ; L^{2}(\mathscr{O})\right)$. This concludes the proof of Lemma 4.1.

For proving our extinction result we need $\mathscr{O} \subset \mathbb{R}$, i.e. $d=1$. To be more specific let $\mathscr{O}=(0, \pi)$. Then $e_{k}(\xi)=\sqrt{\frac{2}{\pi}} \sin k \xi, \quad \xi \in[0, \pi], \lambda_{k}=k^{2}$ and $L^{1}(0, \pi) \subset H$ continuously, so

$$
\gamma=\inf \left\{\frac{|x|_{L^{1}}}{|x|_{-1}}: x \in L^{1}(0, \pi)\right\}>0 .
$$

Theorem 4.2 Let $x \in L^{p}(0, \pi), p \geq \max \{2 m, 4\}$, be such that

$$
|x|_{-1}<C_{N}^{-1} \rho \gamma,
$$

where

$$
C_{N}:=\frac{\pi}{4} \sum_{k=1}^{N}(1+k)^{2} \mu_{k}^{2}
$$

Then, for each $n \in \mathbb{N}$,

$$
\mathbb{P}(\tau \leq n) \geq 1-\frac{|x|_{-1}}{\rho \gamma}\left(\int_{0}^{n} e^{-C_{N} s} d s\right)^{-1},
$$

where by Lemma 4.1 we have

$$
\tau(\omega)=\sup \left\{t \geq 0:|X(t, x)|_{-1}>0\right\} .
$$


Proof. By condition (iv) we see that

$$
r \Psi(r) \geq \rho|r|, \quad \forall r \in \mathbb{R} .
$$

Consider the solution $X_{\lambda} \in L_{W}^{2}\left(0, T ; L^{2}\left(\Omega ; H_{0}^{1}(0, \pi)\right)\right)$ to equation (3.1). Then by first applying Krylov-Rozovskii's Itô formula (cf.[19, Theorem I.3.1] or e.g. [20, Theorem 4.2.5]) and then the classical Itô formula to the real valued semi-martingale $\left|X_{\lambda}(t)\right|_{-1}^{2}, t \in[0, T]$, and the function

$$
\varphi_{\varepsilon}(r)=\left(r+\varepsilon^{2}\right)^{1 / 2}, \quad r \in \mathbb{R},
$$

we find

$$
\begin{aligned}
& d \varphi_{\varepsilon}\left(\left|X_{\lambda}(t)\right|_{-1}^{2}\right)+\left(\left|X_{\lambda}(t)\right|_{-1}^{2}+\varepsilon^{2}\right)^{-1 / 2}\left\langle X_{\lambda}(t), \Psi_{\lambda}\left(X_{\lambda}(t)\right)\right\rangle_{2} d t \\
& =\frac{1}{2} \sum_{k=1}^{N} \mu_{k}^{2} \frac{\left.\left|X_{\lambda}(t) e_{k}\right|_{-1}^{2}\left(\left|X_{\lambda}(t)\right|_{-1}^{2}+\varepsilon^{2}\right)-\left|\left\langle X_{\lambda}(t) e_{k}, X_{\lambda}(t)\right\rangle_{-1}\right|^{2}\right)}{\left(\left|X_{\lambda}(t)\right|_{-1}^{2}+\varepsilon^{2}\right)^{3 / 2}} d t \\
& +\left\langle\sigma\left(X_{\lambda}(t)\right) d W(t), \varphi_{\varepsilon}^{\prime}\left(\left|X_{\lambda}(t)\right|_{-1}^{2}\right) X_{\lambda}(t)\right\rangle \\
& \leq \frac{1}{2} \sum_{k=1}^{N} \mu_{k}^{2} \frac{\left|X_{\lambda}(t) e_{k}\right|_{-1}^{2}}{\left(\left|X_{\lambda}(t)\right|_{-1}^{2}+\varepsilon^{2}\right)^{1 / 2}} d t+\left\langle\sigma\left(X_{\lambda}(t)\right) d W(t), \varphi_{\varepsilon}^{\prime}\left(\left|X_{\lambda}(t)\right|_{-1}^{2}\right) X_{\lambda}(t)\right\rangle \\
& \leq C_{N} \frac{\left|X_{\lambda}(t)\right|_{-1}^{2}}{\left(\left|X_{\lambda}(t)\right|_{-1}^{2}+\varepsilon^{2}\right)^{1 / 2}} d t+2\left\langle\sigma\left(X_{\lambda}(t)\right) d W(t), \varphi_{\varepsilon}^{\prime}\left(\left|X_{\lambda}(t)\right|_{-1}^{2}\right) X_{\lambda}(t)\right\rangle .
\end{aligned}
$$

Here $C_{N}$ is given by (4.12) and

$$
\sigma\left(X_{\lambda}(t)\right) d W(t)=\sum_{k=1}^{N} \mu_{k} X_{\lambda}(t) e_{k} d \beta_{k}(t)
$$

Integrating over $t$ and letting $\lambda \rightarrow 0$ we see that the right hand side of (4.15) converges to the right hand side of (4.16) below in $L^{2}(\Omega ; C([0, T] ; H))$. But by (3.5), (3.8), (3.12), (3.13) and by Proposition 3.4 the same is true for the left hand side with limit

$$
\varphi_{\varepsilon}\left(|X(t)|_{-1}^{2}\right)-\varphi_{\varepsilon}\left(|x|_{-1}^{2}\right)+\int_{0}^{t} \int_{\mathscr{O}} \frac{X(s)}{\left(|X(s)|_{-1}^{2}+\varepsilon\right)^{1 / 2}} \eta(s) d \xi d s .
$$


Taking into account (2.2) and (4.14) we altogether obtain

$$
\begin{aligned}
& d \varphi_{\varepsilon}\left(|X(t)|_{-1}^{2}\right)+\rho \frac{|X(t)|_{L^{1}(0, \pi)}}{\left(|X(t)|_{-1}^{2}+\varepsilon^{2}\right)^{1 / 2}} d t \\
& \leq C_{N} \frac{|X(t)|_{-1}^{2}}{\left(|X(t)|_{-1}^{2}+\varepsilon^{2}\right)^{1 / 2}} d t+2\left\langle\sigma(X(t)) d W(t), \varphi_{\varepsilon}^{\prime}\left(|X(t)|_{-1}^{2}\right) X(t)\right\rangle .
\end{aligned}
$$

Consequently by Lemma 4.1 for all $t \geq 0$

$$
\begin{aligned}
& \varphi_{\varepsilon}\left(|X(t)|_{-1}^{2}\right)+\gamma \rho \int_{0}^{t \wedge \tau} \frac{|X(s)|_{-1}}{\left(|X(s)|_{-1}^{2}+\varepsilon^{2}\right)^{1 / 2}} d s \\
& \leq \varphi_{\varepsilon}\left(|x|_{-1}^{2}\right)+C_{N} \int_{0}^{t \wedge \tau} \frac{|X(s)|_{-1}^{2}}{\left(|X(s)|_{-1}^{2}+\varepsilon^{2}\right)^{1 / 2}} d s \\
& +2 \int_{0}^{t \wedge \tau}\left\langle\sigma(X(s)) d W(s), \varphi_{\varepsilon}^{\prime}\left(|X(s)|_{-1}^{2}\right) X(s)\right\rangle, \quad \mathbb{P} \text {-a.s., }
\end{aligned}
$$

where $\gamma$ is defined by (4.4).

Clearly, we have

$$
\lim _{\varepsilon \rightarrow 0} \int_{0}^{t \wedge \tau} \frac{|X(s)|_{-1}}{\left(|X(s)|_{-1}^{2}+\varepsilon^{2}\right)^{1 / 2}} d s=t \wedge \tau, \quad \mathbb{P} \text {-a.s.. }
$$

Now, letting $\varepsilon$ tend to zero we get

$$
\begin{aligned}
& |X(t)|_{-1}+\gamma \rho(t \wedge \tau) \leq|x|_{-1}+C_{N} \int_{0}^{t}|X(s)|_{-1} d s \\
& +\int_{0}^{t} 1_{[0, \tau]}(s)\left\langle\sigma(X(s)) d W(s), X(s)|X(s)|_{-1}^{-1}\right\rangle \quad \mathbb{P} \text {-a.s. }
\end{aligned}
$$

Hence by a standard comparison result

$$
\begin{aligned}
& |X(t)|_{-1}+\rho \gamma \int_{0}^{t} e^{C_{N}(t-s)} 1_{[0, \tau]}(s) d s \leq e^{C_{N} t}|x|_{-1} \\
& +\int_{0}^{t} e^{C_{N}(t-s)} 1_{[0, \tau]}(s)\left\langle\sigma(X(s)) d W(s), X(s)|X(s)|_{-1}^{-1}\right\rangle .
\end{aligned}
$$


Taking expectation and multiplying by $(\rho \gamma)^{-1} e^{-C_{N} t}$, we obtain

$$
\int_{0}^{t} e^{-C_{N} s} \mathbb{P}(\tau>s) d s \leq \frac{|x|_{-1}}{\rho \gamma} .
$$

Writing $\mathbb{P}(\tau>s)=1-\mathbb{P}(\tau \leq s)$ we deduce that

$$
\mathbb{P}(\tau \leq t) \geq 1-\frac{|x|_{-1}}{\rho \gamma}\left(\int_{0}^{t} e^{-C_{N} s} d s\right)^{-1}
$$

and (4.13) follows.

In particular Theorem 4.2 applies to self-organized criticality stochastic models (1.7)

$$
\left\{\begin{array}{c}
d X(t)-\Delta\left(\rho \operatorname{sign}\left(X(t)-x_{c}\right)+\widetilde{\Psi}\left(X(t)-x_{c}\right)\right) d t \\
\ni \sigma\left(X(t)-x_{c}\right) \sum_{k=1}^{N} \mu_{k} e_{k} d \beta_{k}, \quad t \geq 0, \\
\rho \operatorname{sign}\left(X(t)-x_{c}\right)+\widetilde{\Psi}\left(X(t)-x_{c}\right) \ni 0, \quad \text { on } \partial[0, \pi], \\
X(0, x)=x
\end{array}\right.
$$

Here the function $\widetilde{\Psi}$ is as in assumption (iv) and $x_{c} \in \mathbb{R}$.

Corollary 4.3 Assume that

$$
\left|x-x_{c}\right|_{-1}<\rho \gamma C_{N}^{-1}
$$

where $C_{N}$ is as in (4.12) and $\gamma$ as in (4.11). Then for each $n \in \mathbb{N}$

$$
\mathbb{P}\left(\tau_{c} \leq n\right) \geq 1-\frac{\left|x-x_{c}\right|-1}{\rho \gamma}\left(\int_{0}^{n} e^{-C_{N} s} d s\right)^{-1}
$$

where

$$
\tau_{c}=\inf \left\{t \geq 0:\left|X(t)-x_{c}\right|_{-1}=0\right\}=\sup \left\{t \geq 0:\left|X(t)-x_{c}\right|_{-1}>0\right\} .
$$

and $X=X(t, x)$ is the solution to (4.18) in the sense of Definition 2.1. 
We note that equation (1.7) reduces to (4.18) by shifting the Heavside function with $x_{c}$.

One must notice that if $x>x_{c}$, i.e. if the initial state is in the supercritical region then by positivity result in Theorem 2.2 we have $X(t) \geq x_{c}, \mathbb{P}$-a.s. for all $t \geq 0$. This means that the state remains in the supercritical-critical region for all the time. However, by (4.19) if $\frac{C_{N}|x|_{-1}}{\rho \gamma}$ is small, it reaches the critical state $x_{c}$ with high probability in a finite time i.e. the supercriticalcritical region is completely absorbed by the critical one in a finite time.

Remark 4.4 Let us consider the deterministic case. Then (4.13) implies that $\tau \leq n$ if $n>\frac{|x|_{-1}}{\rho \gamma}$; so $\tau \leq \frac{|x|_{-1}}{\rho \gamma}$. But this, of course, also follows directly from (4.17), since we assume $C_{N}=0$.

Acknowledgement. We would like to thank Philippe Blanchard for introducing us to these models of self-organized criticality and the relevant literature. This work has been supported in part by the CEEX Project 05 of Romanian Minister of Research, the DFG -International Graduate School "Stochastics and Real World Models",the SFB-701 and the BiBoS-Research Center.', the research programme "Equazioni di Kolmogorov" from the Italian "Ministero della Ricerca Scientifica e Tecnologica" and "FCT, POCTI219, FEDER". Most of the work was done during very pleasant visits of the first and second author to the University of Bielefeld and the first and the third author to the SNS in Pisa.

\section{References}

[1] P. Bak, C. Tang and K. Wiesenfeld, Phys. Rev. Lett. 59, 1987, Phys. Rev. A, 38, 1988.

[2] P. Bantay and M. Janosi, Self organization and anomalous diffusions, Physica A, 185, 11-189, 1992.

[3] V. Barbu, Nonlinear semigroups and differential equations in Banach spaces. Noordhoff International Publishing, Leiden, 1976.

[4] V. Barbu, V.I. Bogachev, G. Da Prato and M. Röckner, Weak solution to the stochastic porous medium equations: the degenerate case, J. Functional Analysis, 235 (2), 430-448, 2006. 
[5] V. Barbu and G. Da Prato, The two phase stochastic Stefan problem, Probab. Theory Relat. Fields, 124, 544-560, 2002.

[6] V. Barbu, G. Da Prato and M. Röckner, Existence and uniqueness of nonnegative solutions to the stochastic porous media equation, Indiana University J. Math. (to appear).

[7] V. Barbu, G. Da Prato and M. Röckner, Existence of strong solutions for stochastic porous media equation under general monotonicity conditions, Preprint n.5, SNS Pisa, March 2007.

[8] R. Cafiero, V. Loreto, L. Pietronero, A. Vespignani and S. Zapperi Local Rigidity and Self-Organized Criticality for Avalanches, Europhysics Letters (EPL), 29, n.2, 111-116, 1995.

[9] J.M. Carlson, J.T. Chayes, E.R. Grannan and G.H. Swindle, Selforganized criticality in sandpiles: nature of the critical phenomenon, Phys. Rev. A (3), 42, 2467-2470, 1990.

[10] J.M. Carlson, J.T. Chayes, E.R. Grannan and G.H. Swindle, Selforgainzed criticality and singular diffusion, Phys. Rev. Lett., 65, n.20, 2547-2550, 1990.

[11] G. Da Prato and J. Zabczyk, Ergodicity for infinite dimensional systems, London Mathematical Society Lecture Notes, n.229, Cambridge University, 1996.

[12] A. Díaz-Guilera, Dynamic Renormalization Group Approach to SelfOrganized Critical Phenomena, Europhysics Letters (EPL), 26, n.3, 177182, 1994.

[13] A. Giacometti, and A. Díaz-Guilera, Dynamical properties of the Zhang model of self-organized criticality, Phys. Rev. E, 58, n.1, 247-253, 1998.

[14] G. Grinstein, D.H. Lee and S. Sachdev, Conservation laws, anisotropy, and self-organized criticality in noisy nonequilibrium systems, Phys. Rev. Lett., 64, 1927-1930, 1990.

[15] H.G.E Hentschel and F. Family, Scaling in open dissipative systems, Physical Review Letters, 66, 1982-1985, 1991. 
[16] T. Hwa and M. Kardar, Dissipative transport in open systems: An investigation of self-organized criticality, Phys. Rev. Lett., 62, n.16, 18131816, 1989.

[17] I.M. Janosi, and J. Kertesz, Self-organized criticality with and without conservation, Physica (Amsterdam) A, 200, 179-188 1993.

[18] H. J. Jensen, Self-organized criticality, Cambridge University Press, Cambridge, 1988.

[19] N.V. Krylov and B.L. Rozovskii, Stochastic evolution equations, Translated from Itogi Naukii Tekhniki, Seriya Sovremennye Problemy Matematiki 14(1979), 71-146, Plenum Publishing Corp. 1981.

[20] C. Prevot and M. Röckner, A concise course on stochastic partial differential equations, Lecture Notes in Mathematics, Springer, 2007.

[21] J. Ren, M. Röckner and Feng-Yu Wang, Stochastic generalized porous media and fast diffusions equations, BiBoS-preprint 2006, to appear in J. Diff. Eqn.

[22] D. L. Turcotte, Self-organized criticality, Reports on Progress in Physics, 621, 1377-1429, 1999. 